\section{REVISTA BRASILEIRA DE QUALIDADE DE VIDA}

\title{
Atividade física, imagem corporal e estado nutricional de alunas de dança
}

\author{
Physical activity, body image and nutritional state dance students
}

Tatiane Minuzzi

Universidade Federal de Santa Maria - UFSM - Santa Maria - Rio Grande do Sul - Brasil tatiane.minuzzi@gmail.com

Kelly Christine Maccarini Pandolfo Universidade Federal de Santa Maria - UFSM - Santa Maria - Rio Grande do Sul - Brasil kellypandolfo@hotmail.com

Rafaella Righes Machado Universidade Federal de Santa Maria - UFSM - Santa Maria - Rio Grande do Sul - Brasil rafaellarighes@hotmail.com

Cati Reckelberg Azambuja Universidade Federal de Santa Maria - UFSM - Santa Maria - Rio Grande do Sul - Brasil cati.razambuja@hotmail.com

Daniela Lopes dos Santos Universidade Federal de Santa Maria - UFSM - Santa Maria - Rio Grande do Sul - Brasil lopesdossantosdaniela@gmail.com

\section{RESUMO}

OBJETIVO: Verificar a associação entre o nível de atividade física (NAF), a percepção da imagem corporal, o estado nutricional e o comportamento alimentar de risco em escolares praticantes de dança em uma escola pública do Sul do Brasil.

MÉTODOS: Participaram do estudo 67 estudantes do sexo feminino, com idade média de $15,57 \pm 0,57$ anos, praticantes de dança nas aulas de educação física. O NAF foi avaliado através do IPAQ-versão curta; imagem corporal (IC) através de escala de silhuetas; estado nutricional pelo índice da massa corporal (IMC) e comportamento alimentar de risco através do Eating Attitudes Test (EAT-26).

RESULTADOS: O grupo de estudo apresentou médias de massa corporal de 55,9 $\pm 9,76 \mathrm{~kg}$, estatura de $1,61 \pm 0,06 \mathrm{~m}$, IMC de $21,43 \pm 3,06 \mathrm{~kg} / \mathrm{m}^{2}$, NAF de $517,16 \pm 589,06$ minutos/semana e o

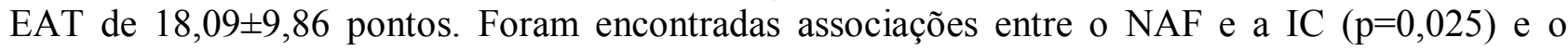
estado nutricional $(\mathrm{p}=0,008)$.

CONCLUSÕES: As adolescentes praticantes de dança do ensino médio apresentaram NAF adequados e que estes se associaram de forma significativa com a IC e o estado nutricional.

PALAVRAS-CHAVE: Atividade física. Imagem corporal. Índice de massa corporal. Estudantes. 


\section{ABSTRACT}

OBJECTIVE: The aim of this study was to investigate the association between physical activity level and body image perception, nutritional status and risky eating behaviors among students who practice dance in a public school in South Brazil.

METHODS: The study included 67 female students with a mean age of $15.57 \pm 0.57$ years, who practiced dance in physical education classes. The PAL was assessed using the short-version of the IPAQ; BI through a silhouette scale, nutritional status by BMI and risk eating behaviors, through the Eating Attitudes Test EAT-26.

RESULTS: The study group showed a mean body mass of $55.9 \pm 9.76 \mathrm{~kg}$, height $1.61 \pm 0.06 \mathrm{~m}$, BMI $21.43 \pm 3.06 \mathrm{~kg} / \mathrm{m}^{2}$, PAL of $517.16 \pm 58906$ minutes / week and EAT $18.09 \pm 9.86$ points Associations were found between the PAL and the BI $(p=0.025)$ and nutritional status $(p=0.008)$.

CONCLUSIONS: We conclude that the high school dance practitioner's adolescents had adequate PAL and that these were associated significantly with the BI and nutritional status.

KEYWORDS: Physical activity. Body image. Body mass index. Students.

\section{Introdução}

O nível de atividade física (NAF) e a prevalência de sedentarismo são estudados em diferentes grupos. Observa-se que as crianças e os adolescentes têm obtido uma atenção especial, pois é nesta fase da vida que hábitos e comportamentos são formados e refletem na idade adulta (AZAMBUJA; PANDOLFO; SANTOS, 2014). Martins (2015) enfatiza que a prática de atividade física pelos jovens, provavelmente, torná-los-ão adultos fisicamente ativos, e uma das formas de promover este estilo de vida em crianças e adolescentes é por meio de programas escolares de educação física.

O hábito de realizar atividade física na adolescência traz vários benefícios para a saúde, como controle e manutenção do peso corporal e redução de riscos cardiovasculares (MORAES et al., 2013). Além desses benefícios, estudos longitudinais evidenciaram que a inatividade física quando iniciada na infância e/ou adolescência tende a continuar na vida adulta e é mais difícil de modificar (AARNIO et al., 2002).

A insatisfação corporal faz parte de um subcomponente da dimensão atitudinal da imagem corporal (IC) e diz respeito à depreciação de sua aparência física (LEPAGE; CROWTHER, 2010). Estudos mostram que a insatisfação corporal está associada com os comportamentos de risco para desencadeamento dos Transtornos Alimentares (ZORDÃO et al., 2015; CUBRELATI et al., 2014; GUIMARÃES et al., 2014). Estudos prévios relatam que a prática da atividade física está associada às melhoras na IC (GONÇALVES; CAMPANA; TAVARES, 2012). Os autores comentam que esse resultado deve-se à obsessão pelas formas corporais magras, o que leva os indivíduos a permanecerem insatisfeitos com a IC.

Outro fator que pode influenciar a IC é o estado nutricional. Segundo Silva et al. (2012), devido à imposição cultural de corpos extremamente magros para o sexo feminino, aqueles indivíduos que não conseguem atingir este padrão corporal estão propensos a desenvolver uma percepção negativa da IC.

Tratando-se de adolescentes do sexo feminino, sabe-se que esta população apresenta menores NAFs quando comparadas a do sexo masculino. Além de diminuir ainda mais com o avanço da idade, o NAF inadequado está associado ao aparecimento de doenças e ao aumento de peso corporal (SEABRA et al., 2008).

Considerando o exposto, o objetivo do presente estudo é verificar a associação entre o NAF, a percepção da IC, o estado nutricional e o comportamento alimentar de risco em escolares praticantes de dança em uma escola pública do Sul do Brasil. 


\section{Métodos}

Participaram do presente estudo 67 escolares do sexo feminino, do ensino médio de uma

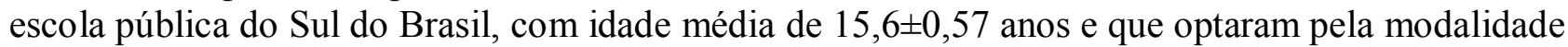
de dança nas aulas curriculares de educação física, no ano de 2014.

O instrumento utilizado para avaliação do NAF foi o Questionário Internacional de Atividades Físicas (IPAQ), versão curta, validado para adolescentes brasileiros por Guedes, Lopes e Guedes (2005). Foram considerados como ativas aquelas com tempo de prática de atividades físicas superior a 300 minutos/semana e como insuficientemente ativas aqueles com tempo inferior a 300 minutos/semana (WORLD HEALTH ORGANIZATION, 2010).

As informações da percepção da IC foram obtidas através da aplicação do instrumento proposto por Tiggemann e Wilson-Barrett (1998), composto por um continuum de nove silhuetas corporais. As adolescentes receberam o instrumento no qual constava as seguintes perguntas: qual silhueta você acha que mais se parece com você? E qual a silhueta que você gostaria de ter? Para verificar a percepção da IC, realiza-se a subtração do número equivalente ao da silhueta real com o da silhueta ideal. Se a variação entre elas for igual à zero, os adolescentes/estudantes são classificados como satisfeitos. Caso a diferença seja positiva, são considerados insatisfeitos pelo excesso de peso (desejo de reduzir a silhueta) e, diferença negativa, insatisfeitos pela magreza (desejo de aumentar a silhueta).

Para determinar o estado nutricional foram coletadas as medidas antropométricas de massa corporal e estatura para posterior cálculo do Índice de Massa Corpórea (IMC). Para aferição da massa corporal utilizou-se balança automática eletrônica, de cristal líquido, plataforma $40 \mathrm{~cm} \mathrm{x}$ $40 \mathrm{~cm}$, capacidade para $150 \mathrm{~kg}$ e fração de $100 \mathrm{gr}$. A estatura foi verificada por meio de estadiômetro, com trena na parede e escala em milímetros. Segundo a classificação da World Health Organization (2010), indivíduos com IMC $\left(\mathrm{kg} / \mathrm{m}^{2}\right)$ :

a) menor que 18,5 apresentam baixo peso;

b) entre 18,5 e 24,9 apresentam peso adequado;

c) entre 25 e 29,9 são pré-obesos;

d) entre 30 e 34,9 tem obesidade classe I;

e) entre 35 e 39,9 tem obesidade classe II;

f) igual ou maior que 40 tem obesidade classe III.

Para fins de categorização, os indivíduos classificados com IMC $\geq 25,0 \mathrm{~kg} / \mathrm{m}^{2}$ foram agrupados em sobrepeso/obesidade.

Para identificar a presença de comportamentos alimentares de risco foi utilizado o teste de atitudes alimentares (Eating Attitudes Test - EAT-26), desenvolvido por Garner e Garfinkel e validado para o português por Bighetti (2003). O EAT-26 é um instrumento com 26 questões em escala Likert, que apresenta 6 opções de resposta: nunca, quase nunca, poucas vezes, às vezes, muitas vezes e sempre. As questões são pontudas de zero a três, sendo que as respostas nunca, quase nunca e poucas vezes somam zero ponto, e as respostas às vezes, muitas vezes e sempre, pontuam 1, 2 e 3 pontos, respectivamente. A pontuação final pode variar de zero a 78 pontos. Na questão 25 , a pontuação é invertida. Indivíduos que atingem até 20 pontos são classificados com comportamento alimentar adequado e, aqueles que somam 21 pontos ou mais, apresentam comportamento alimentar de risco para o desenvolvimento de transtornos alimentares (TA).

As coletas de dados foram realizadas durante os dois tempos consecutivos de aula, destinado à disciplina de educação física curricular do ensino médio na modalidade dança. A primeira coleta realizada foi das medidas antropométricas (peso e estatura) e, logo após, as alunas responderam individualmente os três instrumentos propostos no estudo (IC, NAF e EAT-26).

Como critério de inclusão selecionou-se aquelas estudantes que espontaneamente quiseram participar do estudo e que os pais assinaram o Termo de Consentimento Livre e Esclarecido. 
Análise de dados foi verificada a normalidade dos resultados através do teste KolmogorovSmirnov. Para análise descritiva dos dados foram utilizados frequência, percentual, média e desvio padrão e para a associação entre o NAF e as demais variáveis, o teste Qui-quadrado de Pearson. As análises foram realizadas com o auxílio do Statistical Package for the Social Science, versão 14.0, adotando-se um nível de significância de $p<0,05$.

O presente estudo foi aprovado pelo Comitê de Ética em Pesquisa com Seres Humanos da Universidade Federal de Santa Maria, reconhecida pela Comissão Nacional de Ética em Pesquisa (CONEP/MS), sob o protocolo/CAAE (Certificado de Apresentação para Apreciação Ética) $n^{\circ}$ 0094.0.243.000-11, seguindo o que determina a Declaração de Helsinque, de 1975.

\section{Resultados}

O grupo de estudo apresentou médias de massa corporal de 55,9 $\pm 9,76 \mathrm{~kg}$, estatura de $1,61 \pm 0,06 \mathrm{~m}$, IMC de $21,43 \pm 3,06 \mathrm{~kg} / \mathrm{m} 2$, NAF de $517,16 \pm 589,06$ minutos/semana e o EAT de $18,09 \pm 9,86$ pontos.

A descrição do grupo estudado, estratificado conforme a classificação do NAF, encontra-se nas Tabelas 1 e 2, enquanto que a relação de dependência entre as variáveis NAF, IC, estado nutricional e EAT encontra-se descrita na Tabela 3.

Tabela 1 - Caracterização da amostra de escolares que praticam dança em uma escola pública do Sul do Brasil, estratificados conforme o nível de atividade física

\begin{tabular}{|c|c|c|c|c|c|c|}
\hline \multirow{3}{*}{$\begin{array}{c}\text { Variável } \\
(n=67)\end{array}$} & \multicolumn{6}{|c|}{ Nível de atividade física } \\
\hline & \multicolumn{3}{|c|}{ Insuficientemente Ativo } & \multicolumn{3}{|c|}{ Ativo } \\
\hline & $\mathbf{X} \pm \mathbf{D P}$ & Mínimo & Máximo & $\mathbf{X} \pm \mathbf{D P}$ & Mínimo & Máximo \\
\hline$\overline{\text { Idade }}$ & $15,68 \pm 0,89$ & 14,0 & 17,0 & $15,68 \pm 1,05$ & 14,0 & 17,0 \\
\hline Peso & $53,23 \pm 8,98$ & 39,9 & 78,0 & $57,98 \pm 9,94$ & 44,0 & 90,5 \\
\hline Estatura & $1,61 \pm 0,06$ & 1,50 & 1,70 & $1,61 \pm 0,07$ & 1,48 & 1,76 \\
\hline IMC & $20,40 \pm 3,08$ & 16,39 & 27,69 & $22,19 \pm 2,85$ & 18,78 & 30,24 \\
\hline NAF & $151,96 \pm 77,23$ & 0 & 290 & $768,29 \pm 658,64$ & 305 & 3360 \\
\hline EAT-26 & $16,75 \pm 9,47$ & 3 & 38 & $19,29 \pm 10,05$ & 1 & 50 \\
\hline
\end{tabular}

Fonte: Autoria própria (2015).

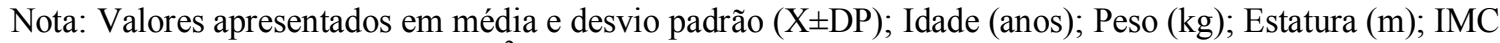
$\left(\mathrm{kg} / \mathrm{m}^{2}\right)$; NAF (minutos/semana); EAT (pontos). 
Tabela 2 - Nível de atividade física estratificado conforme a imagem corporal, estado nutricional e comportamento alimentar de escolares praticantes de dança de uma escola pública do Sul do Brasil

\begin{tabular}{|c|c|c|c|c|c|c|c|}
\hline & \multirow{3}{*}{$\begin{array}{c}\text { Variável } \\
(n=67)\end{array}$} & \multicolumn{6}{|c|}{ Nível de Atividade Física } \\
\hline & & \multicolumn{2}{|c|}{$\begin{array}{c}\text { Insufic. } \\
\text { Ativo }\end{array}$} & \multicolumn{2}{|c|}{ Ativo } & \multicolumn{2}{|c|}{ Total } \\
\hline & & n & $\%$ & n & $\%$ & $\mathbf{n}$ & $\%$ \\
\hline \multirow{4}{*}{$\begin{array}{l}\text { Imagem } \\
\text { Corporal }\end{array}$} & Satisfeito & 8 & 28,6 & 8 & 20,5 & 16 & 25,0 \\
\hline & Insatisfeito p/Magreza & 11 & 39,3 & 6 & 15,4 & 17 & 25,0 \\
\hline & Insatisfeito p/Excesso Peso & 9 & 32,1 & 25 & 64,1 & 34 & 50,0 \\
\hline & Todos & 28 & 100,0 & 39 & 100,0 & 67 & 100,0 \\
\hline \multirow{4}{*}{$\begin{array}{c}\text { Estado } \\
\text { Nutricional }\end{array}$} & Baixo Peso & 8 & 28,6 & 1 & 2,6 & 9 & 13,2 \\
\hline & Peso Adequado & 17 & 60,7 & 34 & 87,2 & 51 & 76,5 \\
\hline & Sobrepeso & 3 & 10,7 & 4 & 10,2 & 7 & 10,3 \\
\hline & Todos & 28 & 100,0 & 39 & 100,0 & 67 & 100,0 \\
\hline \multirow{3}{*}{ EAT-26 } & De Risco & 10 & 35,7 & 18 & 46,2 & 28 & 41,2 \\
\hline & Adequado & 18 & 64,3 & 21 & 53,8 & 39 & 58,8 \\
\hline & Todos & 28 & 100,0 & 39 & 100,0 & 67 & 100,0 \\
\hline
\end{tabular}

Fonte: Autoria própria (2015).

Nota: Insufic. Ativo - Insuficientemente Ativo; Valores apresentados em frequência (n) e percentual (\%); Idade (anos); Peso (kg); Estatura (m); IMC (kg/m²); Nível de Atividade Física (minutos/semana); EAT-26 (pontos).

Tabela 3 - Associação entre nível de atividade física e sexo, imagem corporal, estado nutricional e transtornos alimentares de escolares praticantes de dança de uma escola pública do Sul do Brasil

\begin{tabular}{lc}
\hline Associação & $\boldsymbol{p}$ \\
\hline Nível de Atividade Física x Imagem Corporal & $0,025^{*}$ \\
Nível de Atividade Física x Estado Nutricional & $0,008^{*}$ \\
Nível de Atividade Física x Transtornos Alimentares & 0,393 \\
\hline
\end{tabular}

Fonte: Autoria própria (2015).

As interpretações dos dados dessa relação demonstram não haver associação entre o NAF e o EAT das escolares. Já associações significativas foram verificadas entre o NAF e as variáveis IC $(p=0,025)$ e o estado nutricional $(p=0,008)$. No entanto, foi observado que as associações entre $o$ NAF e a IC e o estado nutricional são fracas, considerando os coeficientes de contingência, $0,315 \mathrm{e}$ 0,356 , respectivamente.

\section{Discussão}

Apesar da importância da prática da atividade física, a incidência de inatividade física na adolescência tem se mostrado alta, repercutindo de forma preocupante, tendo em vista que a prática regular de atividades físicas nesta faixa etária está relacionada à probabilidade de uma vida mais ativa na idade adulta (AZEVEDO et al., 2007). Knuth e Hallal (2009) reafirmam essa preocupação enfatizando que, com o passar dos anos, o número de adolescentes inativos tem aumentado, os quais têm participado cada vez menos nas aulas de educação física e apresentado menores níveis de aptidão física.

Estudo realizado em diversas regiões do Brasil mostrou que na região Sul, 48,4\% dos adolescentes são insuficientemente ativos (HALLAL et al., 2010). O presente estudo verificou que $41,8 \%$ das adolescentes encontravam-se nesta classificação.

A associação entre o NAF e a IC $(\mathrm{p}=0,025)$, assim como o NAF e o estado nutricional $(\mathrm{p}=0,008)$ apresentaram-se significativas. Das escolares praticantes de dança classificadas como ativas, $(58,2 \% ; n=39)$, pode-se observar que $81,7 \%(n=34)$ estavam no peso adequado e, mesmo assim, 64,1\% (n=25), encontravam-se insatisfeitas pelo excesso de peso. Já, entre aquelas classificadas como insuficientemente ativas $(41,8 \%$; $n=28)$, pode-se verificar que $71,4 \%(n=20)$ 
estavam insatisfeitas com sua IC, apesar de 60,7\% $(n=17)$ destas estarem com o peso adequado (Tabela 2).

No estudo realizado por Lara et al. (2014) foi verificado que também houve grave distorção da IC em escolares que, mesmo estando com a massa corporal adequada, estavam insatisfeitas com o seu peso. Em estudo realizado por Azambuja, Pandolfo e Santos (2014), avaliando a insatisfação com a IC de 115 escolares do $8^{\circ}$ ano do Ensino Fundamental de uma escola pública federal de Santa Maria, RS, foi encontrando em $64,2 \%$ das meninas o desejo de modificar a silhueta corporal. Da mesma forma, Glaner et al. (2013), em estudo com 637 adolescentes de escolas públicas do município de Saudades, SC, encontraram prevalência de insatisfação corporal em 65,2\%.

De acordo com Pinheiro e Giugliani (2006), a forma e o esquema corporal, muitas vezes, levam à insatisfação com o próprio corpo, mesmo com dimensões corporais dentro de parâmetros adequados para esse grupo populacional e para a saúde. Ao estratificar a insatisfação corporal quanto ao desejo de aumentar a silhueta (insatisfeitas pela magreza) ou o desejo de diminuir a silhueta (insatisfeitas pelo excesso de peso), independente da classificação do NAF, observou-se que metade das adolescentes $(\mathrm{n}=34)$ pesquisadas gostaria de diminuir a silhueta corporal. Estes resultados corroboram com outros estudos (PINHEIRO; GIUGLIANI, 2006; RIBEIRO; VEIGA, 2010; REIS et al., 2014) nos quais a distorção da imagem tende sempre pela busca de um corpo mais magro. Um recente estudo realizado por Zordão et al. (2015), que procurou investigar a insatisfação com a IC e transtornos alimentares em adolescentes de Minas Gerais, mostrou que grande parte das adolescentes que compuseram a amostra do estudo considerou um corpo magro como ideal. Pelo menos metade das meninas relatou possuir o desejo de estar conforme a silhueta 3 , idealizada como magra.

Entre aquelas que manifestaram o desejo de aumentar a silhueta corporal, estavam as que apresentavam baixo peso e as eutróficas, representando $25,0 \%$ do total da amostra. No estudo realizado por Fidelix et al. (2011), ao dicotomizar a insatisfação com a IC em desejo de reduzir e em desejo de aumentar o tamanho da silhueta corporal, foi observado que $26,5 \%$ das meninas apresentavam o desejo de aumentar o tamanho corporal.

Em relação ao estado nutricional, independente da classificação do NAF, observou-se que $76,5 \%(n=51)$ estavam com peso adequado. Já no estudo realizado por Pelegrini e Petroski (2009), adolescentes do sexo feminino que apresentaram baixo peso tinham maior risco de serem insuficientemente ativas, ao contrário do que a maior parte dos estudos vem demonstrando, onde o sobrepeso e a obesidade são fortemente associados à inatividade física (MITCHELL et al., 2013; CABRERA et al., 2014). Portanto, pode-se inferir que na tentativa de reduzir os sentimentos de insatisfação corporal, adolescentes possam praticar exercícios físicos além dos níveis considerados adequados para a saúde.

Em relação ao comportamento alimentar, não foi encontrada associação significativa com o NAF. Contudo, conforme a Tabela 2, 41,2\% $(\mathrm{n}=28)$ das escolares apresentaram comportamento alimentar de risco. Este dado, segundo Cubrelati et al. (2014), deve ser visto com atenção pela comunidade escolar, tendo em vista estudos apontarem que a adolescência é um período de risco para o desenvolvimento de transtornos alimentares e as meninas estão mais predispostas a esses distúrbios. Sendo assim, redobrar os cuidados quanto às medidas preventivas e de intervenção no sentido de conscientizar as adolescentes sobre a importância de hábitos alimentares saudáveis que faz muito necessário.

Os números encontrados para o comportamento alimentar de risco foram superiores aos achados em outro estudo com adolescentes brasileiras. Dunker, Fernandes e Carreira Filho (2009), ao investigarem a prevalência de sintomas de anorexia e bulimia em 183 adolescentes da cidade de São Paulo, encontraram índices de $21,1 \%$ para o risco de desenvolvimento de transtornos alimentares.

Considera-se a percepção de IC um fator de risco que não se deve ignorar e possui origem na tendência socialmente imposta sobre o ideal de magreza e da boa forma física, especialmente às mulheres, nas quais a aparência física representa uma importante medida de valor pessoal (AZAMBUJA et al., 2014). 
Conclui-se que adolescentes do sexo feminino, praticantes de dança, do ensino médio de uma escola pública do Sul do Brasil, apresentaram níveis de atividade física adequados e que estes se associaram de forma significativa com a IC e o estado nutricional, mas não se associaram com o comportamento alimentar de risco. Portanto, torna-se necessário maior atenção em relação ao comportamento das escolares, buscando prevenir possíveis distorções da IC, visto que muitos dos hábitos e dos comportamentos estabelecidos na adolescência são, possivelmente, transferidos para a idade adulta, tornando-se mais difíceis de serem modificados.

Entre as limitações deste estudo encontram-se o baixo número amostral, com coleta de dados em corte transversal, não possibilitando o acompanhamento longitudinal das adolescentes e a relação causa-efeito entre as variáveis.

\section{Agradecimentos}

Agradecemos ao Colégio Militar de Santa Maria, RS, Brasil, o qual nos proporcionou e auxiliou no desenvolvimento deste estudo.

\section{Referências}

AARNIO, M., WINTER, T.; PELTONEN, J.; KUJALA, U. M.; KAPRIO, J. Stability of leisuretime physical activity during adolescence - a longitudinal study among 16, 17 and 18 years-old Finnish youth. Scandinavian Journal of Medicine \& Science in Sports, v. 12, n. 3, p. 179-185, 2002. crossef

AZAMBUJA, C. R.; PANDOLFO, K. C. M.; SANTOS, D. L. Autopercepção da imagem corporal e estado nutricional de escolares. Revista Brasileira de Nutrição Esportiva, v. 8, n. 47, p. 316-321, 2014.

AZAMBUJA, C. R.; PANDOLFO, K. C. M.; BRUM, L. M.; SANTOS, D. L.; SCHETINGER, M. R. C. Educação em ciências: a influência do estilo de vida dos adolescentes de escolas públicas federais frente às ações preventivas de saúde. Revista Ciências \& Ideias, v. 5, n. 2, p. 81-99, 2014.

AZEVEDO, M. R.; ARAUJO, C. L.; COZZENSA DA SILVA, M.; HALLAL, P. C. Tracking of physical activity from adolescence to adulthood: a population-based study. Revista Saúde Pública, v. 41, n. 1, p. 69-75, 2007. Crossef

BIGHETTI, F. Tradução e validação do Eating Attitudes Test (EAT-26) em adolescentes do sexo feminino na cidade de Ribeirão Preto - SP. 2003. 123 f. Dissertação (Mestrado em Enfermagem em Saúde Pública) - Universidade de São Paulo, Ribeirão Preto, 2003.

CABRERA, T. F. C.; CORREIA, I. F. L.; SANTOS, D. O. D.; PACAGNELLI, F. L.; PRADO, M. T. A.; SILVA, T. D. D.; FERNANI, D. C. G. L. Análise da prevalência de sobrepeso e obesidade e do nível de atividade física em crianças e adolescentes de uma cidade do sudoeste de São Paulo.

Revista Brasileira de Crescimento e Desenvolvimento Humano, v. 24, n. 1, p. 67-72, 2014.

CUBRELATI, B. S.; RIGONI, P. A. G.; VIEIRA, L. F.; BELEM, I. C. Relação entre distorção de imagem corporal e risco de desenvolvimento de transtornos alimentares em adolescentes.

Conexões: Revista da Faculdade de Educação Física da UNICAMP, v. 12, n. 1, p. 1-15, 2014. 
DUNKER, K. L. L.; FERNANDES, C. P. B.; CARREIRA FILHO, D. Influência do nível socioeconômico sobre comportamentos de risco para transtornos alimentares em adolescentes. Jornal Brasileiro de Psiquiatria, v. 58, n. 3, p. 156-161, 2009. Crossef

FIDELIX, Y. L., SILVA; D. A. S.; PELEGRINI, A.; SILVA, A. F. D.; PETROSKI, E. L. Insatisfação com a imagem corporal em adolescentes de uma cidade de pequeno porte: associação com sexo, idade e zona de domicílio. Revista Brasileira de Cineantropometria e Desempenho Humamno, v. 13, n. 3, p. 202-207, 2011.

GLANER, M. F.; PELEGRINI, A.; CORDOBA, C. O.; POZZOBON, M. E. Associação entre insatisfação com a imagem corporal e indicadores antropométricos em adolescentes. Revista Brasileira de Educação Física e Esporte, v. 27, n. 1, p. 129-136, 2013. rossef

GONÇALVES, C. D. O.; CAMPANA, A. N.; TAVARES, M. D. C. Influência da atividade física na imagem corporal: uma revisão bibliográfica. Motricidade, v. 8, n. 2, p. 70-82, 2012. Crossef

GUEDES, D. P.; LOPES, C. C.; GUEDES, J. E. R. P. Reprodutibilidade e validade do Questionário Internacional de Atividade Física em adolescentes. Revista Brasileira de Medicina e Esporte, v. 11, n. 2, p. 151-158, 2005. Crossef

GUIMARÃES, A. D.; MACHADO, S. P.; FRANÇA, A. K. T. D. C.; CALADO, I. L. Transtornos alimentares e insatisfação com a imagem corporal em bailarinos. Revista Brasileira de Medicina do Esporte, v. 20, n. 4, p. 267-271, 2014. Crossef

HALLAL, P. C.; KNUTH, A. G.; CRUZ, D. K. A.; MENDES, M. I.; MALTA, D. C. L. Prática de atividade física em adolescentes brasileiros. Ciência \& Saúde Coletiva, v. 15, n. 2, p. 3035-3042, 2010. crossef

KNUTH, A. G.; HALLAL, P. C. Temporal trends in physical activity: a systematic review. Journal of Physical Activity \& Health, v. 6, n. 5, p. 548-559, 2009.

LARA, S.; COPETTI, J.; LANES, K. G.; PUNTEL, R. L.; FOLMER, V. Imagem corporal, medidas antropométricas e atividade física como ferramentas para a educação em saúde no contexto escolar. Revista Ciências \& Ideias, v. 4, n. 2, p. 27-46, 2014.

LEPAGE, M. L.; CROWTHER, J. H. The effects of exercise on body satisfaction and affect. Body Image, v. 7, n. 2, p. 124-130, 2010. Crossef

MARTINS, J. F. S. F. Educação física e estilos de vida: porque são os adolescentes fisicamente (in) ativos?. 2015. 371 f. Tese (Doutorado em Ciências da Educação na Especialidade de Didática da Educação Física e Desporto) - Universidade de Lisboa, Lisboa, 2015.

MITCHELL, J. A.; RODRIGUEZ, D.; SCHMITZ, K. H.; AUDRAIN-MCGOVERN, J. Greater screen time is associated with adolescent obesity: a longitudinal study of the BMI distribution from ages 14 to 18 . Obesity, v. 21, n. 3, p. 572-575, 2013. crossef

MORAES, A C. F.; CARVALHO, H. B.; REY-LÓPEZ, J. P.; GRACIA-MARCO, L.; BEGHIN, L.; KAFATOS, A.; MORENO, L. A. Independent and combined effects of physical activity and sedentary behavior on blood pressure in adolescents: gender differences in two cross-sectional studies. PLoS One, v. 8, n. 5, May 2013. crossef 
PELEGRINI, A.; PETROSKI, E. L. Inatividade física e sua associação com estado nutricional, insatisfação com a imagem corporal e comportamentos sedentários em adolescentes de escolas públicas. Revista Paulista de Pediatria, v. 27, n. 4, p. 366-373, 2009. Crossef

PINHEIRO, A. P.; GIUGLIANI, E. R. J. Body dissatisfaction in Brazilian school children: prevalence and associated factors. Revista de Saúde Pública, v. 40, n. 3, p. 489-496, 2006. Crossef

REIS, N.; MACHADO, Z.; PELEGRINI, A.; MONTE, F.; BOING, L.; SIMAS, J.; GUIMARÃES, A. Imagem corporal, estado nutricional e sintomas de transtornos alimentares em bailarinos.

Revista Brasileira de Atividade Física \& Saúde, v. 18, n. 6, p. 771-781, 2014.

RIBEIRO, L. G.; VEIGA, G. V. Imagem corporal e comportamentos de risco para transtornos alimentares em bailarinos profissionais. Revista Brasileira de Medicina do Esporte, v. 16, n. 2, p. 99-102, 2010. Crossef

SEABRA, A. F.; MENDONÇA, D. M.; THOMIS, M. A.; ANJOS, L. A.; MAIA, J. A.

Determinantes biológicos e sócio-culturais associados à prática de atividade física de adolescentes Biological and socio-cultural determinants of physical activity in adolescents. Caderno de Saúde Pública, v. 24, n. 4, p. 721-736, 2008. Crossef

SILVA, J. D.; SILVA, A. B. D. J.; OLIVEIRA, A. V. K. D.; NEMER, A. S. D. A. Influência do estado nutricional no risco para transtornos alimentares em estudantes de nutrição. Ciência da Saúde Coletiva, v. 17, n. 12, p. 3399-3406, 2012. Crossef

TIGGEMANN, M.; WILSON-BARRETT, E. Children's figure ratings: relationship to self-esteem and negative stereotyping. International Journal of Eating Disorders, v. 23, n. 1, p. 83-88, 1998. crossef

WORLD HEALTH ORGANIZATION. Global recommendations on physical activity for health. Geneva, 2010. Disponível em:

http://www.who.int/dietphysicalactivity/factsheet_recommendations/en/. Acesso em: 05 out. 2014.

ZORDÃO, O. P.; BARBOSA, A.; PARISI, T. S. A.; GRASSELLI, C. D. S. M.; NOGUEIRA, D. A.; SILVA, R. R. Associação da imagem corporal e transtornos alimentares em adolescentes de Minas Gerais (Brasil). Nutrición Clínica y Dietética Hospitalaria, v. 35, n. 2, p. 48-56, 2015.

\section{Conflitos de interesse}

Não há nenhum potencial conflito de interesse entre os autores desse trabalho. 